\title{
The Structure of the $\mathrm{NH}_{2}$ Group in Some Amides and Sulfamates from Proton Magnetic Resonance Spectra of Powders
}

\author{
B J ØR N PEDERSE N
}

\author{
Sentralinstitutt for industriell forskning, Oslo 3, Norway
}

\begin{abstract}
The proton magnetic resonance spectra of thirteen powdered amides and sulfamates have been recorded. Using least squares methods a theoretical line shape has been fitted to each of the experimental spectra, and the obtained agreement is in all, but one case, very good.

From the line shape analysis the proton-proton distance, $R$, within the $\mathrm{NH}_{2}$ group in each compound is determined. Assuming an $\mathrm{N}-\mathrm{H}$ bond distance of $1.03 \AA$, the obtained values of $R$ show that the $\mathrm{H}-\mathrm{N}-\mathrm{H}$ angle in the amides, except sulfamide, is about $120^{\circ}$ and in the sulfamates and sulfamide about $110^{\circ}$.
\end{abstract}

The least squares method recently developed to analyse the line shape of 1 the proton magnetic resonance spectra of pair-powders ${ }^{1}$ has been applied to spectra of compounds containing $\mathrm{NH}_{2}$ groups, and the results will be presented here. We want to discuss to what extent (1) the least square fitted spectra are in agreement with the observed spectra, (2) the physical significance of the derived parameters on which the calculated line shape is based, and (3) the obtained structural information on the shape of the $\mathrm{NH}_{2}$ group.

\section{BACKGROUND}

A nitrogen atom forming three bonds can be found in two extreme, ideal configurations: trigonal pyramidal or trigonal planar. The $-\mathrm{NH}_{2}$ group is generally assumed to be pyramidal in amines and planar in amides. ${ }^{2}$ Microwave studies of some simple amides have shown, however, that the $-\mathrm{NH}_{2}$ group is not exactly planar in these compounds, but deviates somewhat from planarity. ${ }^{3}$ The question then arises whether there is a tendency for the $-\mathrm{NH}_{2}$ group to be either planar or pyramidal, or if the group always can be regarded as more or less pyramidal, changing shape with its bonding situation. A recent neutron diffraction study of potassium sulfamate ${ }^{4}$ has shown

Acta Chem. Scand. 22 (1968) No. 6 
that the $-\mathrm{NH}_{2}$ group is pyramidal in the sulfamate ion. This is contrary to what one would predict from the fairly short $\mathbf{S}-\mathbf{N}$ bond length indicating some double bond character. ${ }^{5}$ Hence, at present it seems uncertain to use the $\mathrm{X}-\mathrm{N}$ bond length to infer the shape of the $-\mathrm{NH}_{2}$ group.

From proton magnetic resonance spectra of solid compounds containing $-\mathrm{NH}_{2}$ groups, we can not obtain the detailed geometry of the whole group. The only parameter we can determine is the $\mathrm{H}-\mathrm{H}$ distance, $R$. But this distance will be a sensitive measure of the "flatnes" of the $\mathrm{NH}_{2}$ group as $R$ will increase with the increase in the $\mathrm{H}-\mathrm{N}-\mathrm{H}$ angle from $109.5^{\circ}$ (ideal pyramidal) to $120^{\circ}$ (ideal planar).

The difficulty of growing sufficiently large single crystal has limited us to study the compounds as powders. From a line shape analysis of the proton magnetic resonance spectra we can deduce two parameters $\alpha$ and $\beta^{2}$ where

$$
\begin{aligned}
& \alpha=3 \mu /\left(2 R^{3}\right) \\
& \beta^{2}=M_{2 \mathrm{e}}
\end{aligned}
$$

Here $\mu$ is the magnetic dipole moment of a proton, and $M_{2 \mathrm{e}}$ the interpair contribution to the Van Vleck second moment.1

The line shape analysis is performed by fitting a theoretical line shape to the experimental spectrum by the method of least squares. ${ }^{1}$ The theoretical line shape is applicable to the analysis of NMR-spectra of powders where the resonant nuclei are situated in magnetically equivalent pairs which are weakly coupled to each other. Hence, this analysis can be used in the analysis of spectra of powders where all hydrogen atoms are located in crystallographically equivalent $-\mathrm{NH}_{2}$ groups. $\alpha$ is a measure of the dipolar coupling within the pair, and $\beta$ is a measure of the dipolar coupling between the pairs and to other magnetic nuclei in the sample. The theoretical line shape is believed to be a better representation of the spectrum the smaller the ratio $\beta / \alpha$, and the analysis seems to break down for values of $\beta / \alpha$ larger than 0.5 . This line shape analysis has been applied earlier in the analysis of some hydrates, ${ }^{1}$ perhydrates, ${ }^{6}$ and potassium bicarbonate, ${ }^{7}$ and this work must be regarded as a part of a general effort to explore the applicability of this method of line shape analysis going on in our laboratory.

From eqn. (1) we see that $\alpha$ is directly related to $R$, the $\mathrm{H}-\mathrm{H}$ distance in the $-\mathrm{NH}_{2}$ group. Using a $\mathrm{N}-\mathrm{H}$ distance of $1.03 \AA$ (vide infra) we find that $\alpha$ will be equal to $3.6 \mathrm{G}$ for the ideal planar configuration and $4.5 \mathrm{G}$ for the ideal pyramidal configuration, i.e. the expected variation in $\alpha$ is $0.9 \mathrm{G}$, which is about twenty times the experimental uncertainty in $\alpha$.

The value of $\beta^{2}$ also obtained from the spectral analysis contains additional structural information. However, $\beta^{2}$ is not very susceptible to minor changes in the structural arrangement, making it useless as a source of information on the shape of the $-\mathrm{NH}_{2}$ group. We will therefore in this study only compare the experimental $\beta^{2}$ with the calculated $M_{2 \mathrm{e}}$ as a check to see if the line shape analysis contains serious systematic errors influencing the experimental value of $\alpha$. 


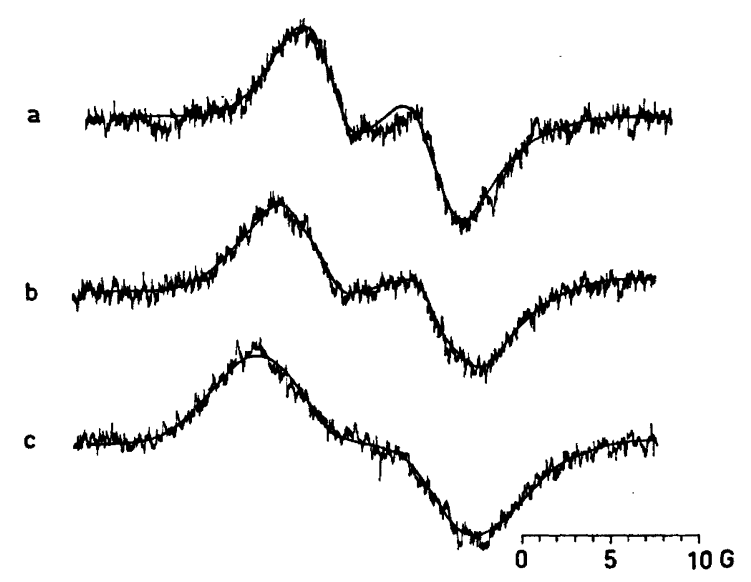

Fig. 1. The proton magnetic resonance spectra of powdered a) potassium oxamate, b) oxamide and c) urea. The calculated spectra are drawn as smooth curves.

\section{EXPERIMENTAL DETAILS}

All amides studied, except potassium oxamate, are commercially available, and they have been used as received. The sulfamates were made by neutralisation of a solution of sulfamic acid with the metal carbonate or hydroxide. Potassium oxamate monohydrate was made in a similar way from oxamic acid. The solvent was partially evaporated at room temperature, and the crystallized salt filtered off. The salt was dehydrated at about $100^{\circ}-150^{\circ} \mathrm{C}$. X-Ray Debye-Scherrer powder diagrams were taken of all salts and compared with literature data.

The proton magnetic resonance spectra were recorded on a Dual Purpose NMR Spectrometer from Varian Asc. operating at $60 \mathrm{MHz}$ in a low proton background probe (V 4331 LP). The magnetic field was swept with a Fieldial unit also from Varian Asc. The scanning speed was from $0.3 \mathrm{G} / \mathrm{min}$ to $2.5 \mathrm{G} / \mathrm{min}$ with corresponding time constants from $12 \mathrm{sec}$ to $0.8 \mathrm{sec}$. Maximum r.f. amplitude was $1 \mathrm{mG}$ or less depending on the sample.

About five spectra of each compound were analysed. A theoretical line shape was fitted to the experimental observed one by least squares methods as discussed in detail elsewhere. 1 Representative experimental and calculated spectra of all compounds are shown in Figs. 1, 2, and 3. The values of $\alpha$ and $\beta / \alpha$ obtained from the analyses are given in Tables 2 and 3 . The values of $\beta^{2}$ are corrected for modulation broadening. ${ }^{1}$

\section{DISCUSSION}

The structure of the $-\mathrm{NH}_{2}$ group in several powdered amides and amines has been studied earlier by Kromhoult and Moulton. ${ }^{8}$ They derived the total second moment from the experimental spectra, and compared this experimental value with the theoretical second moment calculated from Van Vleck's formula. The information on the shape of the $\mathrm{NH}_{2}$ group was obtained from that part of the experimental second moment which remained when the interpair part and the part due to the coupling to the nitrogen atom were subtracted. All the experimental uncertainty was then collected in $R$, leading to a fairly inaccurate determination. Furthermore, as found by Smith and Cotts, ${ }^{9}$ and recently discussed in detail by Leppelmeyer and Hahn, ${ }^{10}$

Acta Chem. Scand. 22 (1968) No. 6 


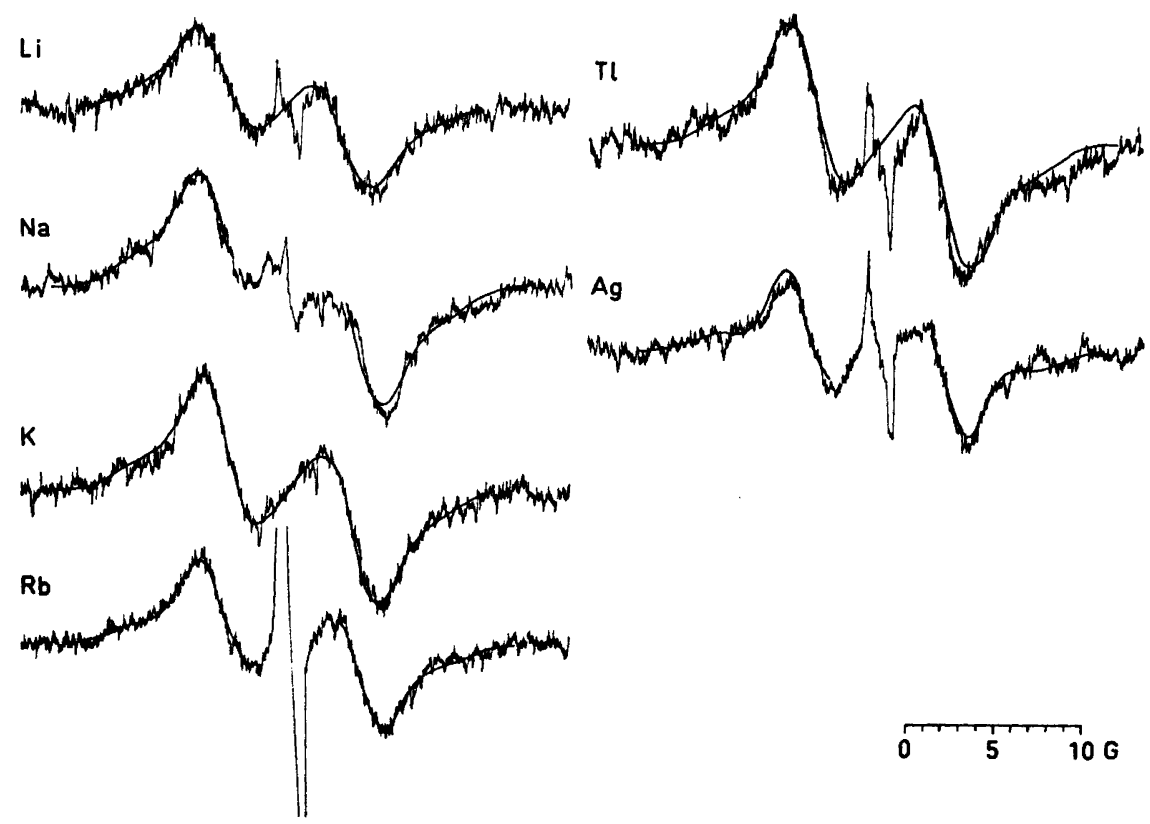

Fig. 2. The proton magnetic resonance spectra of powdered sulfamates with monovalent cations, $\mathrm{MeSO}_{3} \mathrm{NH}_{2}$. The calculated spectra are drawn as smooth curves.

the Van Vleck second moment formula only gives the upper limit to the contribution from the proton-nitrogen coupling. A reduction in the $\mathrm{N}-\mathrm{H}$ contribution has also been found experimentally by Emsley and Smith ${ }^{11}$ in thiourea. The values of $R$ deduced from difference second moments as used by Kromhoult and Moulton, ${ }^{8}$ are therefore systematically too large. The valuable aspect of the line shape analysis performed here is that the intrapair, $0.8 \alpha^{2}$, and the interpair part, $\beta^{2}$, of the second moment are obtained separately. A closely related way of analysing the spectra of $\mathrm{NH}_{2}$ groups has earlier been used by Freeman and Richards ${ }^{12}$ in their study of potassium amide, and by Richards and York ${ }^{13}$ in their study of nitroguanidine.

From the spectra in Figs. 1, 2, and 3 it can be seen that the fitted theoretical spectra are in remarkably good agreement with the experimental spectra. The central peak observed in some of the spectra is due to occluded water in the sample, and this part of the curve has not been used in the analysis. The largest deviation between the calculated and observed spectrum is seen in the spectrum of calcium sulfamate in Fig. 3. In this salt the values of the intrapair and the interpair coupling seem to be coupled in such a way that the interpair coupling is small when the intrapair coupling is large and vice versa. The tails of the spectrum are therefore better resolved than the central part, and it seems impossible to fit a theoretical spectrum, based on an isotropic $\beta^{2}$, to the experimental spectrum. This spectrum, therefore, is not used in the subsequent analysis. 
Fig. 3. The proton magnetic resonance spectra of powdered sulfamates with divalent cations, $\mathrm{Me}\left(\mathrm{SO}_{3} \mathrm{NH}_{2}\right)_{2}$. The calculated spectra are drawn as smooth curves.
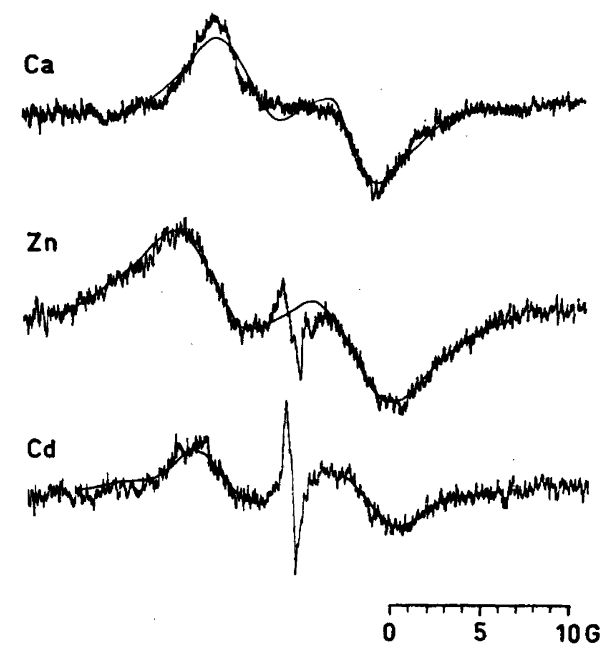

It is an open question to what extent the derived values of $\alpha$ and $\beta$ are coupled so that each will be systematically shifted away from the true value even in the cases where the agreement between the theoretical and experimental spectra is good. It is clear that when the experimental and the theoretical spectrum are in close agreement the second moment of the experimental spectrum must be equal to the second moment of the theoretical spectrum: $0.8 \alpha^{2}+\beta^{2}$. It is therefore reasonable to think that in general $\alpha$ and $\beta$ will be coupled in such a way that if $\beta$ is found too small, $\alpha$ will be found too large. We can therefore use the relation $\beta^{2}=M_{2 \mathrm{e}}$ to test the result of the line shape analysis, as experience shows that $M_{2 \mathrm{e}}$ is fairly insensitive to minor changes in the atomic positions. ${ }^{6}$ In Table 1 are given the observed $\beta^{2}$ and the calculated $M_{2 \mathrm{e}}$ from the Van Vleck formula in the cases where the crystal structure is known. In urea ${ }^{14}$ and potassium sulfamate ${ }^{4}$ the proton positions are known from neutron diffraction studies. The proton positions in oxamide have been inferred from the positions of the heavier atoms ${ }^{15}$ by placing the $\mathrm{H}$-atoms in the molecular plane, symmetrically about

Table 1. Calculated, $M_{2 \mathrm{e}}$, and observed, $\beta^{2}$, values of the interpair part of the second moment.

\begin{tabular}{lcccccc}
\hline & $\beta^{2}(\mathrm{G})$ & $M_{2 \mathrm{e}}\left(\mathrm{G}^{2}\right)$ & $M_{2}\left(\mathrm{G}^{2}\right)$ & $\alpha^{\prime}(\mathrm{G})$ & $\alpha(\mathrm{G})$ & $\beta / \alpha$ \\
\hline Urea & $5.81(10)$ & $7.5+k \cdot 1.8=8.4$ & 21.9 & 4.11 & 4.48 & 0.54 \\
Oxamide & $3.73(10)$ & $5.3+k \cdot 1.8=6.2$ & 17.7 & 3.79 & 4.19 & 0.46 \\
$\begin{array}{l}\text { Potassium } \\
\text { sulfamate }\end{array}$ & $1.49(4)$ & $0.77+k \cdot 1.8=1.7$ & 15.9 & 4.21 & 4.24 & 0.33 \\
\hline
\end{tabular}

Acta Chem. Scand. 22 (1968) No. 6 
the $\mathrm{C}-\mathrm{N}$ direction, $1.03 \AA$ away from $\mathrm{N}$, and with the angle $\mathrm{H}-\mathrm{N}-\mathrm{H}$ equal to $120^{\circ}$.

As pointed out above, Leppelmeyer and Hahn ${ }^{10}$ have found that the $\mathrm{N}-\mathrm{H}$ contribution to the second moment is reduced from the Van Vleck value which is $1.8 \mathrm{G}^{2}$ (assuming the $\mathrm{N}-\mathrm{H}$ distance equal to $1.03 \AA$ ). The reduction factor $k$ in Table 1 has been found by these authors to depend on the orientation of the external magnetic field relative to the principal axes of the electric field gradient at the nitrogen site. The reduction factor is $\left(1+v_{\mathrm{q}}^{2} / v_{1}^{2}\right)^{-1}$, where $v_{1}$ is the larmor frequency in a given field, and $v_{\mathrm{q}}$ the pure quadrupole resonance frequency as observed when the magnetic field is directed along one of the principal axes. In our experiments the external field is about 14 $k \mathrm{G}$, hence $v_{1}=4.3 \mathrm{MHz} . v_{\mathrm{q}}$ in different nitrogen containing compounds has been found to lie between 2 and $4.6 \mathrm{MHz},{ }^{16}$ i.e. approximately equal to $v_{1}$. We will therefore assume that $k \approx 0.5$. Using this value we can calculate $M_{2 \mathrm{e}}$ as done in Table 1.1

As can be seen in Table $1, M_{2 \mathrm{e}}$ is much larger than $\beta^{2}$ in urea and oxamide, and approximately equal to $\beta^{2}$ in potassium sulfamate. This indicates that the parameters derived from the line shape analysis of urea and oxamide are not reliable. This is probably due to the large values of $\beta / \alpha$ in these compounds telling that the pairs are fairly strongly coupled contrary to the idea behind the theoretical line shape analysis. ${ }^{1}$ In Table 1 is also calculated the intrapair coupling, $\alpha^{\prime}$, from the difference $M_{2}-M_{2 \mathrm{e}}$. $\alpha^{\prime}$ derived for urea, $4.11 \mathrm{G}$, is smaller, than $\alpha$, but still higher than the value observed in a single crystal study, 3.61 G. ${ }^{17}$ This is difficult to understand, but our value for $M_{2}, 21.9 \mathrm{G}^{2}$, is somewhat larger than a value found earlier, ${ }^{8} 20.8 \pm 0.6 \mathrm{G}^{2}$. Also in oxamide the difference between $\alpha$ and $\alpha^{\prime}$ is significant showing that the line shape analysis contains systematic errors in this case too. Our value of $M_{2}, 17.7 \mathrm{G}^{2}$, however, is in good agreement with an earlier value, ${ }^{8} 17.5 \pm 0.8 \mathrm{G}^{2}\left(\mathrm{at}-196^{\circ} \mathrm{C}\right)$. In potassium oxamate the difference between $\alpha$ and $\alpha^{\prime}$ is only $0.03 \mathrm{G}$, which is insignificant. In lack of more detailed information, we will assume that the reason why the line shape analysis breaks down for urea and oxamide is the large value of $\beta / \alpha$. In all other compounds we have studied, the value of $\beta / \alpha$ is smaller, and we will take the analysis of the potassium sulfamate data as a

Table 2. Experimental values of $\alpha$ and $\beta / \alpha$ in amides and derived molecular parameters.

\begin{tabular}{llcccc}
\hline Compound & $\begin{array}{c}\alpha \\
(\mathrm{G})\end{array}$ & $\begin{array}{c}\mathrm{R} \\
(\AA)\end{array}$ & $\begin{array}{c}r\left(\varphi=120^{\circ}\right) \\
(\AA)\end{array}$ & $\begin{array}{c}\varphi(r=1.03 \AA) \\
\left({ }^{\circ}\right)\end{array}$ & $\beta / \alpha$ \\
\hline Potassium oxamate & 3.59 & 1.806 & 1.043 & 122.5 & 0.39 \\
Urea & $3.61^{a}$ & 1.803 & 1.041 & 122.2 & 0.54 \\
Oxamide & $3.8^{b}$ & 1.77 & 1.02 & 118.4 & 0.46 \\
Nitroguanidin & $3.86^{c}$ & 1.762 & 1.017 & 117.6 & 0.63 \\
\hline
\end{tabular}

a Ref. 17. $\quad b \alpha^{\prime} . \quad c$ Ref. 13. 
support for the physical significance of $\alpha$ and $\beta$ derived from the line shape analysis of the spectra of sulfamates.

Potassium sulfamate has also been studied in single crystal and powdered form by Easwaran. ${ }^{18}$ Somewhat surprisingly his value of the second moment of the powder spectrum, $23.9 \pm 1.0 \mathrm{G}^{2}, \alpha, 4.83 \mathrm{G}$, and $\beta^{2}, 5.2 \mathrm{G}^{2}$, are much larger than our values. But his powder spectrum is very similar to the spectrum obtained here, and its shape is not in accordance with the fairly large $\beta / \alpha$ ratio, 0.47 , calculated from his values of $\alpha$ and $\beta$.

\section{The intrapair coupling constant, $\alpha$}

In Table 2 are collected the different experimental values of $\alpha$ in amides and different molecular parameters derived from $\alpha$.

From the value of $\alpha$ we can determine the $\mathrm{H}-\mathrm{H}$ distance, $R . R$, in turn, is determined by the $\mathrm{N}-\mathrm{H}$ distance, $r$, and the $\mathrm{H}-\mathrm{N}-\mathrm{H}$ angle, $\varphi$, but it is impossible to decompose $R$ into unambiguous values of $r$ and $\varphi$. In Table 2 are also given the values of these parameters by first calculating $r$ from $R$ setting $\varphi=120^{\circ}$, and then calculating $\varphi$ by setting $r=1.03 \AA$.

It is reasonable to assume that the $\mathrm{N}-\mathrm{H}$ distance will not change by more than $0.01 \AA$ with a change in the geometry of the $\mathrm{NH}_{2}$ group from planar to pyramidal, as have been found for carbon compounds. ${ }^{19}$ Furthermore, the fairly long hydrogen bonds formed by $\mathrm{NH}_{2}$ groups will not affect the $\mathrm{N}-\mathrm{H}$ distance much. The expected variation in the hydrogen bonding from one compound to the other will, therefore, probably not introduce a significant change in the $\mathrm{N}-\mathrm{H}$ distance. We have chosen the value $1.03 \AA$ for the $\mathrm{N}-\mathrm{H}$ distance. This is the mean of the values found by setting $\varphi=120^{\circ}$, and receives support from the neutron diffraction investigation of urea by Worsham, Levy and Peterson ${ }^{14}$ who find $\varphi=122.1^{\circ}$. However, this should not been pushed too far, O'Reilly and Tsang ${ }^{20}$ using lattice harmonics have reanalysed

Table 3. Experimental values of $\alpha$ and $\beta / \alpha$ in sulfamates and derived molecular parameters.

\begin{tabular}{lccccc}
\hline Compound & $\begin{array}{c}\alpha \\
(G)\end{array}$ & $\begin{array}{c}R \\
(\AA)\end{array}$ & $\begin{array}{c}r\left(\varphi=109.5^{\circ}\right) \\
(\AA)\end{array}$ & $\begin{array}{c}(r=1.03 \AA) \\
\left({ }^{\circ}\right)\end{array}$ & $\beta / \alpha$ \\
\hline Li-sulfamate & 4.02 & 1.740 & 1.065 & 115.2 & 0.375 \\
Na-sulfamate & 4.29 & 1.702 & 1.042 & 111.4 & 0.385 \\
K-sulfamate & 4.24 & 1.709 & 1.047 & 112.1 & 0.288 \\
Rb-sulfamate & 4.41 & 1.686 & 1.032 & 109.4 & 0.281 \\
Tl-sulfamate & 4.33 & 1.697 & 1.039 & 111.0 & 0.314 \\
Ag-sulfamate & 4.45 & 1.682 & 1.020 & 109.5 & 0.256 \\
Ca-sulfamate & 3.90 & & & & 0.378 \\
Zn-sulfamate & 4.73 & 1.648 & 1.009 & 106.2 & 0.452 \\
Cd-sulfamate & 4.79 & 1.640 & 1.004 & 105.6 & 0.300 \\
sulfamide & 4.36 & 1.693 & 1.040 & 110.6 & 0.435 \\
K-amide ${ }^{a}$ & 4.86 & 1.632 & 0.999 & 104.8 & 0.515 \\
\hline
\end{tabular}

$a$ Ref. 12. e.s.d. in $\alpha: 0.03 G$ and $\beta / \alpha: 0.005$; hence e.s.d. in $R: 0.003 \AA, r: 0.002 \AA$ and $\varphi: 0.2^{\circ}$.

Acta Chem. Scand. 22 (1968) No. 6 
the data of Andrew and Hyndman, ${ }^{17}$ and concluded that the two $\mathrm{N}-\mathrm{H}$ distances are not equal, and they find $r=1.024 \pm 0.017 \AA$ and $1.085 \pm 0.026 \AA$ and $\varphi=116 \pm 4^{\circ}$.

The experimental $\alpha$-values for the sulfamates, sulfamide and $\mathrm{K}$-amide are given in Table 3 together with derived molecular parameters. As the neutron diffraction study of potassium sulfamate ${ }^{4}$ showed that the $\mathrm{NH}_{2}$ group was pyramidal with an HNH angle close to the tetrahedral angle, the value of $r$ has been calculated for this value of $\varphi$. Again the mean NH distance derived is close to $1.03 \AA$. If we use this value of $r$, we can see from Table 3 that $\varphi$ is about $110^{\circ}$ for the monovalent salts, only the Li-salt exhibits an angle somewhat larger, and in the divalent salts the angle appears to be smaller than the tetrahedral angle. In the sulfamates the cations are very likely coordinated to the oxygen atoms. ${ }^{4}$ The smaller cations excert a stronger polarizing force on the anions than the larger cations. It might then be that the increasing value of $\alpha$ with the increasing radius of the cation, as evident from the data in Table 3, is a measure of this polarizing effect, as one would expect that a polarized sulfamate ion will have a less pyramidal $\mathrm{NH}_{2}$ group.

We see also from the data in Table 3 that the value of $\alpha$ for sulfamide shows that the $\mathrm{NH}_{2}$ group in this compound has about the same shape as in the sulfamate ion. Hence, despite the shorter $\mathrm{N}-\mathrm{S}$ distance in this compound, 1.600 (9) $\AA,{ }^{21}$ compared to the corresponding distance in potassium sulfamate, 1.666 (6) $\AA, 4$ the shape of the $-\mathrm{NH}_{2}$ group does not seem to be affected.

The value of $\alpha$ determined for potassium amide by Freeman and Richards 12 (see Table 3) also fits into this picture. With a value of $r=1.03 \AA$ we find a value of $\varphi=104.8^{\circ}$ indicating that the $\mathrm{NH}_{2}^{-}$ion, which is isoelectronic with the water molecule, also has the same shape as the water molecule.

In the above discussion we have not taken into account the effect of motion of the $\mathrm{NH}_{2}$ group on the values of $\alpha$ and $\beta^{2}$. The observed $\alpha$ and $\beta^{2}$ will always be averaged over the intramolecular and librational vibration. Detailed NMR studies of hydrates have shown that for the $\mathrm{H}_{2} \mathrm{O}$ molecules the motion reduces the value of $\alpha$ with about $18 \%$ from the value calculated using the equilibrium dimensions of the isolated $\mathrm{H}_{2} \mathrm{O}$ molecule. ${ }^{22}$ However, the variation in $\alpha$ from one hydrate to another, as observed at room temperature, is much smaller, only about $3 \%{ }^{23}$ If the same applies to the $-\mathrm{NH}_{2}$ group the observed variation in $\alpha$ observed here is much too large to orginate from the difference in motion from one compound to another. It is difficult to calculate quantitatively the motional correction for the $\mathrm{NH}_{2}$ group in the different compounds as it requires detailed information on the nature of the motion. ${ }^{24}$ However, as the $-\mathrm{NH}_{2}$ group is part of a molecule or an ion it is expected that the motional correction will be smaller here than for the $\mathrm{H}_{2} \mathrm{O}$ molecule with its small moments of inertia. Furthermore, some of the motional correction will be taken up by the selected value of the $\mathrm{N}-\mathrm{H}$ bond length, $1.03 \AA$, which, therefore, is not directly comparable to $\mathrm{N}-\mathrm{H}$ distances determined by other techniques. ${ }^{19}$

Acknowledgement. This work has been supported by The Royal Norwegian Council for Scientific and Industrial Research. We would like to thank Mrs. M. Jørve for skillful technical assistance, and various members of the staff at the Institute for valuable discussions. 


\section{REFERENCES}

1. Pedersen, B. Acta Chem. Scand. 22 (1968) 444.

2. Wells, A. F. Structural Inorganic Chemistry, Clarendon Press, Oxford 1962, Ch. XVI, p. 604.

3. Costain, C. C. and Dowling, J. M. J. Chem. Phys. 32 (1960) 158; Randall, E. W. and Baldeschweiler, J. D. J. Mol. Spectry. 8 (1962) 365.

4. Cox, G. W., Sabine, T. M., Padmanabhan, V. M., Bau, N. T., Chung, M. K. and Surjadi, A. J. Acta Cryst. 23 (1967) 578.

5. Jeffrey, G. A. and Stadler, H. P. J. Chem. Soc. 19511467.

6. Pedersen, B. F. and Pedersen, B. Selected Topics in Structure Chemistry, Universitetsforlaget, Oslo 1967.

7. Pedersen, B. Acta Cryst. B 24 (1968) 471.

8. Kromhoult, R. A. and Moulton, W. G. J. Chem. Phys. 23 (1955) 1673; Multon, W. G. and Kromhoult, R. A. J. Chem. Phys. 25 (1956) 34.

9. Smith, D. H. and Cotts, R. M. J. Chem. Phys. 41 (1964) 2403.

10. Leppelmeyer, G. W. and Hahn, E. L. Phys. Rev. 141 (1966) 724.

11. Emsley, J. W. and Smith, J. A. S. Trans. Faraday Soc. 57 (1961) 1233.

12. Freeman, R. and Richards, R. E. Trans. Faraday Soc. 52 (1956) 802.

13. Richards, R. E. and York, R. W. Trans. Faraday Soc. 54 (1958) 321.

14. Worsham, J. E., Levy, H. A. and Peterson, S. W. Acta Cryst. 10 (1957) 319.

15. Ayerst, E. M. and Duke, J. R. C. Acta Cryst. 7 (1954) 588.

16. Abe, Y. J. Phys. Soc. Japan 23 (1967) 51.

17. Andrew, E. R. and Hyndman, D. Discussions Faraday Soc. 19 (1955) 195.

18. Easwaran, K. R. K. Indian J. Pure Appl. Phys. 4 (1966) 324.

19. Sutton, L. E. Tables of Interatomic Distances and Configuration in Molecules and Ions, Suppl. 1956-59, Chem. Soc. London 1965.

20. O'Reilly, D. E. and Tsang, T. Phys. Rev. 128 (1962) 2639.

21. Trueblood, K. N. and Mayer, S. W. Acta Cryst. 9 (1956) 628.

22. Pedersen, B. J. Chem. Phys. 41 (1964) 122.

23. Pedersen, B. Unpublished results.

24. Ibers, J. A. and Stevenson, D. P. J. Chem. Phys. 28 (1958) 929.

Received January 8, 1968. 